\title{
ReiDoCrea, un proyecto de innovación en la transparencia docente
}

\section{ReiDoCrea, an Innovation Project on Transparency in Teaching}

José Miguel García Ramírez. Universidad de Granada

Recepción: 12 de enero de 2015 | Revisión: 15 de enero de 2015 | Aceptación/Publicación: 21 de enero de 2015 Evaluado por: Marzia Fiorini. Universitá di Cassino | Karen McMullin. Trent University

Correspondencia con el autor: miguelgr@ugr.es

\section{Resumen}

La calidad y excelencia docente en la Educación Superior implican un alto nivel de competencias tanto en el profesorado como en el alumnado (Healey, 2000). El proyecto de innovación docente de la Universidad de Granada "ReiDoCrea" se adscribe al modelo de Excelencia Visible (Bolívar y Caballero, 2010) y utiliza la metodología de Investigación-Acción. Método: Participaron 553 estudiantes ( $M=26.4$; $S D=5.8$ ), durante el curso académico 1012-13-14, elaborando artículos de investigación y contestando un cuestionario de pregunta abierta. Resultados: Se publican 51 artículos $(n=208$ | 37.5\%) de 159 artículos presentados. Las categorías emergentes significativas por puntos fuertes, débiles y posibilidad de mejora (Atla.ti): Motivación $(\mathrm{f}=90 \%)$, Satisfacción $(\mathrm{f}=88 \%)$, Dificultad $(\mathrm{f}=95 \%)$ y Formación $(\mathrm{f}=99 \%)$. Conclusiones: Necesidad de implementar un programa de orientación y formación en la elaboración de un estudio de investigación.

Palabras clave: Educación | Creatividad | Innovación | Empatía | Motivación

\begin{abstract}
Quality and excellence in teaching in Higher Education imply a high level of competencies both in the professors and in the students (Healey, 2000). ReiDoCrea, an innovation project of the University of Granada is based on the Visible Excellence model (Bolívar y Caballero, 2010) and uses Action-Research methodology. Method: 553 students participated in the study $(M=26.4 ; S D=5.8)$ during the academic year 1012-13-14, writing research articles and answering an open-question questionnaire. Results: 51 articles ( $n=208 \mid 37.5 \%$ students) of 159 papers presented were published. Significant emerging categories for strengths, weaknesses and potential for improvement (Atla.ti) points: Motivation ( $f=90 \%)$, Satisfaction $(f=88 \%)$, Difficulty $(f=95 \%)$ and Training $(f=99 \%)$. Conclusions: Need to implement an orientation and training program in the development of a research study.
\end{abstract}

Keywords: Education | Creativity | Innovation | Empathy | Motivation

\section{Introducción}

La calidad y excelencia docente en la Educación Superior implica un alto nivel de competencias tanto en el profesorado como en el alumnado (Healey, 2000). La profesión de profesorado universitario tiene como componentes: la docencia, la investigación, la innovación y la gestión. El componente que ocupa mayor parte del tiempo es la docencia, aunque institucionalmente se da más importancia a la 
investigación. De todas maneras, la docencia debe considerarse unida a los demás componentes, como un todo, de forma equitativa para evitar que se suplanten unas a otras (Garcia-Ramirez, 2014; Shulman, 2004) caracterizada por objetivos, preparación, métodos utilizados, resultados significativos, visibilidad de la enseñanza, interacción comunicativa y reflexión crítica (Glassick, Taylor y Maeroff, 2003).

La universidad tiende a la garantía de la calidad y excelencia visible de la docencia, o sea a una enseñanza orientada al estudiante, pública, susceptible de revisión crítica y accesible (Bolívar y Caballero, 2008; Shulman, 2004); siendo claves la comunicación y la interacción social en el proceso de enseñanza-aprendizaje para desarrollar las relaciones interpersonales (Goleman, 2006), a partir de la empatía como proceso de escucha que permite comprender a las demás personas poniéndose en su lugar sin necesidad de juzgar ni necesariamente dar la razón; por lo tanto, a través de la empatía el profesorado puede desarrollar habilidades pedagógicas que le permiten transmitir su conocimiento y reducir los efectos negativos de la escucha selectiva (Garcia-Ramirez y Perez-Villen 2003; Garcia-Ramirez, 2012); también la empatía permite reducir los efectos causados por la estima y las exigencias psicológicas, factores psicosociales implícitos a la docencia (Garcia-Ramirez, 2014; Codina, 2004).

La innovación docente es uno de los componente clave en mejora la calidad de la Educación Superior, ya que favorece e incrementa la eficiencia y la satisfacción en el proceso de enseñanza-aprendizaje; además es necesaria para motivar y desarrollar la creatividad tanto en el profesorado como en el alumnado. Además, la sociedad demanda una enseñanza creativa, interdisciplinar y contextualizada. Por lo tanto, a través de la innovación docente las universidades pueden cumplir con la responsabilidad social de ofrecer una enseñanza de calidad y transparente, de carácter público (Garcia-Ramirez, 2011, 2012, 2013, 2014; Fiorini y Garcia-Ramirez, 2013; Garcia-Ramirez, Garcia-Sempere y Fiorini, 2012; Ruiz y Garcia-Ramirez, 2005; Diaz, Vazquez, \& McMullin, 2014).

El proyecto de innovación docente ReiDoCrea que se desarrolla e implementa en la Universidad de Granada, a través de una revista electrónica de investigación en la que los estudiantes publican los trabajos de investigación que realizan durante el curso académico (Garcia-Ramirez, 2012, 2013, 2014). Este proyecto de innovación docente se adscribe al modelo de Excelencia Visible por su carácter publico, social y colaborativo de la construcción del conocimiento adquirido (Bolívar y Caballero, 2008). También se utiliza la metodología de Investigación-Acción, para generar un cambio de actitud y comportamiento, promocionar el aprendizaje social y cooperativo, y potenciar el desarrollo personal de identidad sociocultural; posibilitando el cumplimiento de los objetivos marcados por el proyecto: Mejorar la docencia, como proceso de enseñanzaaprendizaje; Incrementar la satisfacción y la motivación por competencias y de logro tanto en docentes como en estudiantes; Aumentar la motivación en la educación universitaria; Desarrollar un nuevo instrumento de evaluación del rendimiento académico; divulgar el conocimiento adquirido por el alumnado; y promover la transparencia docente (Garcia-Ramirez, 2014).

\section{Método}

\section{Participantes}

Participaron 553 estudiantes, 378 mujeres y 175 hombres, con edades comprendidas entre 20 y 67 años $(M=26.4, S D=5.8)$. Los participantes, voluntario, eran estudiantes de los grados de Pedagogía (20\%), Primaria (20\%), Educación Social $(20 \%)$, Psicología (20\%), Logopedia (2\%), Máster (9\%), Doctorado (7\%) y Posdoctoral $(2 \%)$. 


\section{Procedimiento e instrumentos}

Durante los cursos académicos 2012-13-14 los/as participantes tuvieron que realizar un estudio de investigación y transcribirlo en forma de artículo dentro de su programa de académico. Los artículos tenían que cumplir con las normas de publicación de la revista ReiDoCrea. Después de que los estudios fueran evaluados por el profesorado participante, estos los remitían a la revista para su posible publicación; luego los/as participante tenían que completar un cuestionario de una pregunta abierta en el que se le pedía que describiera su experiencia, expresando los puntos fuerte, débiles y posibilidad de mejora. Por lo tanto, se pretendió medir la percepción de logro; entendiendo como logro la publicación del estudio de investigación. La evaluación fue por pasos de manera sistemática: $1^{\mathrm{a}}$. Evaluación positiva por el profesorado participante; $2^{\mathrm{a}}$. Valoración positiva de los trabajos por los evaluadores externos; $3^{\mathrm{a}}$. Completar un cuestionario de pregunta abierta (máximo de 200 palabras), explicando su experiencia. Los datos obtenidos se analizaron a través del programa Atla.ti para poder codificar el material y describir las categorías emergentes.

\section{Resultados}

Los/as 553 participantes presentaron 159 artículos valorados positivamente por su profesorado. El 99\% de los/as participantes superaron la evaluación de las asignaturas adscritas al proyecto ReiDoCrea. De los 159 artículos, presentados en ReiDoCrea, fueron evaluados positivamente y publicados 51, elaborados por 208 participantes, este resultado equivale al $37.5 \%$ de los artículos presentados; siendo publicados en dos volúmenes (Garcia-Ramirez, 2013, 2014).

Después se procedió a realizar el análisis del cuestionario para conocer los puntos fuertes, débiles y posibilidades de mejora como categorías emergentes independientes, considerando la frecuencia (f) de cada una en el tanto por ciento que le correspondiese siempre fuera superior al $70 \%$ (Atla.ti). En esta valoración se le pedió al estudiante participante que describiera su experiencia, expresando como se sintió durante todo el proceso de elaboración, revisión y publicación de su trabajo. Los/as participantes entregaron el cuestionario completado, después de saber que artículos fueron evaluados positivamente para su publicación. El cuestionario fue completado por el $75 \%$ de los participantes. Las categorías emergentes significativas como puntos fuertes fueron: Motivación ( $f=90 \%$ ), publicación del artículo ( $f=88 \%$ ) y satisfacción (80\%). La categorías emergentes como puntos débiles fueron: Redactar el artículo $(\mathrm{f}=95 \%)$ y revisión del artículo $(71 \%)$. Las categorías emergentes como posibilidades de mejora fueron: Formación para realizar un estudio de investigación $(f=99 \%)$ y el feedback ( $f=71 \%$ ). Los puntos débiles y la posibilidad de mejora se deben principalmente a la inseguridad y falta de apoyo en el momento de transcribir su estudio de investigación, principalmente, porque no tuvieron formación previa de cómo elaborar un artículo científico.

A continuación se exponen en la siguiente tabla:

\begin{tabular}{|c|c|c|}
\hline \multicolumn{3}{|c|}{$\begin{array}{c}\text { Tabla } 1 \\
\text { Categorías emergentes }\end{array}$} \\
\hline PUNTOS FUERTES & PUNTOS DÉBILES & POSIBILIDADES DE MEJORA \\
\hline Motivación $\mathrm{f}=90 \%$ & & \\
\hline Publicación & Redactar & Formación \\
\hline Satisfacción $\mathrm{f}=80 \%$ & Revisión & Feedback \\
\hline
\end{tabular}




\section{Discusión y conclusiones}

Los resultados obtenidos justifican la necesidad de potenciar la innovación docente como una de las parte que junto a la enseñanza, la investigación y la gestión constituyen la profesión de docente universitario. También los resultados muestran que hay que insistir en la importancia de comunicación entre docentes y estudiantes como clave para cumplir con la responsabilidad social de ofrecer una enseñanza de calidad. El feedback es necesidad que el análisis muestra y que el alumnado necesita para formarse adecuadamente.

La innovación docente como parte integrante del proceso de enseñanza aprendizaje ayuda al profesorado a conocer los puntos débiles y de esa forma poder incluir en el programa las mejoras necesarias para que su alumnado adquiera y desarrolle tanto los conocimiento como las competencias necesarias para sentir satisfacción y eficiencia de su periodo formativo. La innovación docente ayuda tanto al profesorado como a los estudiantes a construir el conocimiento de manera social o colaborativa y desde la empatía como proceso de escucha. Por lo tanto, ReiDoCrea es un proyecto de innovación docente, con el cual se puede dar el reconocimiento a los trabajos realizados por el alumnado lo que está relacionado significativamente con la motivación por competencias y de logro (García-Ramírez, 2014).

Para concluir, con ReiDoCrea no sólo se posibilitado la construcción de conocmiento colaborativamente si no que se ha facilitado la divulgación del conocimiento adquirido respondiendo a la demanda social de transparencia docente; además de ser un vehículo de reconocimiento y eficiencia de una Educación de Calidad y Excelencia Visible (Bolívar y Caballero, 2008), que partiendo de los recursos existentes se ha creado algo nuevo para resolver un problema o falta de transparencia o visibilidad en la docencia universitaria (Fiorini y García-Ramírez, 2013); pero aun así sigue siendo necesario implementar un plan de formación especifico en investigación, tal como mostró el análisis de los datos obtenidos del cuestionario.

\section{Referencias}

Bolívar, A., Caballero, K. (2008). Cómo hacer visible la excelencia en la enseñanza universitaria. Revista Iberoamericana de Educación. (OEI). $n^{\circ}$ 46/8,1-10.

Codina, A. (2004). Saber escuchar. Un intangible Valioso. Intangible Capital, 4, 1-26. http://www.intangiblecapital.org/index.php/ic/article/view/23/29

Diaz-Marin, V., Vazquez-Martinez, A.I., \& McMullin, K.J. (2014). First steps towards a university social network on personal learning environments. The International Review of Research in Open and Distance Learning, 15(3), 93-119. http://www.irrodl.org/index.php/irrodl/article/view/1679/2910

Fiorini, M.; García-Ramírez, JM. (2013). Cap. 5: Técnicas de grupo y creatividad aplicadas en el ámbito universitario,117-147. En Villena Martínez, MD.; Muñoz García, A. (2013). Recursos para la tutoría en el aula universitaria. Granada: Editorial Universidad de Granada. http://www.worldcat.org/title/recursos-para-latutoria-en-el-aula-universitaria/oclc/870115874

Garcia-Ramirez, J.M. (2014). Selección de indicadores para la evaluación de la excelencia docente en la Universidad de Trent (Canadá). Granada: Universidad de Granada, 2014. http://hdl.handle.net/10481/30350 
Garcia-Ramirez, J.M. (2012). La comunicación, clave de excelencia visible en la Educación Superior. Journal for Educators, Teachers and Trainers, 3, 25-36. http://hdl.handle.net/10481/22300

Garcia-Ramirez, J.M. (2011). Una reconsideración de la excelencia visible en la educación superior: la escucha empática. Andaluciaeduca, 66, 84. http://www.andaluciaeduca.com/hemeroteca/ae_digital66.pdf

Garcia-Ramirez, J.M. (2012). Las Tecnologías de la Información y la Comunicación, TIC, en la educación universitaria. Andaluciaeduca, 76, 77. http://www.andaluciaeduca.com/hemeroteca/ae_digital76.pdf

Garcia-Ramirez, J.M. (2012). Docencia-Creativa: una estrategia de motivación a través de la difusión del conocimiento. Reidocrea, 1, 1-3. http://hdl.handle.net/10481/33571

Garcia-Ramirez, J.M. (2013). ReiDoCrea un proyecto de construcción y divulgación del conocimiento. Reidocrea, 2, 1-10. http://hdl.handle.net/10481/34149

Garcia-Ramirez, J.M. (2014). ReiDoCrea, un proyecto de investigación-acción. Reidocrea, 3, 1-3. http://hdl.handle.net/10481/34151

Garcia-Ramirez, J.M.; García-Sempere, P.J.; Fiorini, M. (coords.). (2012). Docencia universitaria y creatividad. Granada: Universidad de Granada. http://hdl.handle.net/10481/33193

Garcia-Ramirez, J.M. (ed.). (2012). ReiDoCrea: volumen 1. Granada: Universidad de Granada. http://hdl.handle.net/10481/33702

Garcia-Ramirez, J.M. (ed.). (2013). ReiDoCrea: volumen 2. Granada: Universidad de Granada. http://hdl.handle.net/10481/34150

Garcia-Ramirez, J.M. (ed.). (2014). ReiDoCrea: volumen 3. Granada: Universidad de Granada. http://hdl.handle.net/10481/34152

Garcia-Ramirez, J.M.; Perez-Villen, MP. (2003). Educación y convivencia, 555-557. En Alvaraz-Rodriguez, J.; Casares-Garcia, P.; Luengo-Navas, J. (2003). Participación, Convivencia y Ciudadanía. Granada: Ediciones Osuna.

Glassick, C., Taylor, M., Maeroff, G. (2003). La valoración del trabajo académico. México D.F.: ANUIES.

Goleman, D. (2006). Inteligencia Social. La nueva ciencia para mejorar las relaciones humanas. México D.F.: Planeta.

Healey, M. (2000). Developing the Scholarship of Teaching in Higher Education: A discipline-based approach. Higher Education Research \& Development, Vol. $19, \mathrm{n}^{\circ} 2$, pp. 169-189.

Shulman, L.S. (2004). Teaching as Community Property: Essays on Higher Education. Ed., Pat Hutchings. San Francisco: Jossey-Bass. 